\title{
Specific clinical and immune features of CD68 in glioma via I,024 samples
}

This article was published in the following Dove Press journal: Cancer Management and Research

\author{
Le Wang ${ }^{1,2, *}$ \\ Chaoqi Zhangl,3,* \\ Zhen Zhang' \\ Bing $\mathrm{Han}^{4}$ \\ Zhibo Shen' \\ Lifeng $\mathrm{Li}^{1,3}$ \\ Shasha Liu' \\ Xuan Zhao' \\ Fanglei $\mathrm{Ye}^{2}$ \\ Yi Zhang ${ }^{1,3,5}$
}

'Biotherapy Center, The First Affiliated Hospital of Zhengzhou University, Zhengzhou, Henan 450052, China; ${ }^{2}$ Department of Otology, The First Affiliated Hospital of Zhengzhou University, Zhengzhou, Henan 450052,

China; ${ }^{3}$ Cancer Center, The First Affiliated Hospital of Zhengzhou University, Zhengzhou, Henan 450052, China; ${ }^{4}$ Department of General ICU, The First Affiliated Hospital of Zhengzhou University, Zhengzhou, Henan 450052, China; ${ }^{5}$ Henan Key Laboratory for Tumor Immunology and Biotherapy, Zhengzhou, Henan 450052, China

*These authors contributed equally to this work

Correspondence: Yi Zhang

Biotherapy Center, The First Affiliated Hospital of Zhengzhou University, I Jianshe East Road, Zhengzhou, Henan 450052, China

Tel/fax +86 37I 66295320

Email yizhang@zzu.edu.cn
Background: There is a growing recognition that tumor-associated macrophages (TAMs) are recruited to the glioma environment, facilitating tumor proliferation and migration by creating an immunosuppressive microenvironment. CD68 has been widely reported as a specific marker of TAMs in cancer.

Purpose: To clarify the role of CD68 in glioma, we investigated its function at the transcriptome level and relationship with clinical practice.

Patients and methods: In total, 325 RNA-seq data from Chinese Glioma Genome Atlas (CGGA) and 697 RNA-seq data from The Cancer Genome Atlas (TCGA) network were enrolled in this study. CD68-specific findings were further analyzed with $\mathrm{R}$ language, and the prognostic impacts were validated through analyzing the overall survival (OS).

Results: CD68 showed a positive correlation with the WHO grade of malignancy in glioma. Meanwhile, CD68 was predominantly expressed in IDH wide type and mesenchymal subtype. Gene ontology (GO) analysis revealed that CD68-related genes were closely related to inflammatory response and immune response. Moreover, seven cultures of metagenes further confirmed that CD68 was a specific marker for macrophages in inflammatory response and played an important role in suppressing T-cell-mediated immunity. The Pearson correlation test suggested that CD68 showed robust correlation with other markers of macrophages and immune checkpoints, including PD-1 and TIM-3. Clinically, a high expression level of CD68 in tumors exhibited a poor survival in glioma patients.

Conclusion: Our results demonstrated that CD68 acted as an immune suppressor and contributed to glioma progression in the tumor microenvironment. These findings may expand our understanding of CD68-specific clinical and immune features in glioma.

Keywords: CD68, tumor-associated macrophage, glioma, immunotherapy

\section{Introduction}

Glioma accounts for $\sim 50 \%$ of all primary brain tumors and is thus the most common intrinsic brain tumors in humans. ${ }^{1}$ Conventional treatment for gliomas consists of surgical resection followed by radiotherapy and chemotherapy. ${ }^{2}$ Despite the improvements in standard treatment, patients who suffer from the most aggressive type, glioblastoma (GBM), have a median survival time of only 14-15 months. ${ }^{3}$ Given these clinical challenges of glioma, there is considerable interest in whether immune mechanisms may have therapeutic value. It has been demonstrated that tumor microenvironment plays a critical role in supporting the malignant growth and progression of glioma. ${ }^{4,5}$ Tumor microenvironment in the brain is composed of various non-neoplastic cell types, including fibroblasts, infiltrating immune cells, and endothelial cells. These stromal cell types interact with tumor cells to produce cytokines, chemokines, and angiogenic molecules. ${ }^{6,7}$ 
Evidence from clinical and experimental studies indicates that tumor-associated macrophages (TAMs) are enriched in glioma and associated with poor prognosis. ${ }^{8}$ Moreover, TAMs are a critical roadblock to the development of cancer immunotherapies. Therefore, therapies targeted against the TAMs may represent a promising approach for treating glioma. ${ }^{9}$

Macrophages have been functionally categorized into M1 and M2 polarized cells that correspond to proinflammatory and anti-inflammatory responses, respectively. In line with their functions, TAMs are generally characterized as M2-like macrophage phenotype in tumor microenvironment. ${ }^{10}$ In GBM, M2 TAMs have been mainly described for their immunosuppressive and tumor-supportive functions. ${ }^{11}$ Despite that TAMs were reported to play an important role in glioma, the molecular characteristics underlying the protumorigenic functions of TAMs remain to be elucidated.

TAMs are commonly identified by expression of CD68, CD163 or CD11b. ${ }^{12}$ Recently, a study showed that the accumulation of $\mathrm{CD} 68^{+} / \mathrm{CD} 11 \mathrm{~b}^{+} / \mathrm{CD} 163^{+} \mathrm{M} 2$ TAMs in patients with glioma was closely correlated with poor prognosis. ${ }^{11}$ Targeting surface markers such as CD11b by using Mac-1 antibodies had been investigated in the tumor mouse model. ${ }^{13}$ Our previous study also showed that Pseudomonas aeruginosa-mannose-sensitive hemagglutinin re-educated $\mathrm{CD} 163^{+}$ M2 macrophages to M1 macrophages in vitro. ${ }^{14} \mathrm{CD} 68^{+} \mathrm{TAMs}$ are also associated with decreased survival rates in patients with cancers, including breast cancer and ovarian cancer. ${ }^{15,16}$ However, there are limited studies about the key molecular characteristics and clinical applications of CD68.

In this study, we aimed to investigate the role of CD68 mRNA expression in whole WHO grade glioma. To explore the expression state of CD68 in glioma, we took advantage of 325 RNA-seq data from Chinese Glioma Genome Atlas (CGGA) dataset. Furthermore, the RNA-seq data of 697 gliomas obtained from The Cancer Genome Atlas (TCGA) network were used to validate our finding. This is the first integrative study characterizing CD68 expression in whole grade glioma molecularly and clinically. A better understanding of the CD68 state and feature in glioma will surely help to further optimize associated cancer therapies.

\section{Methods}

\section{Patients and data collection}

CD68 expression data of gliomas were obtained from CGGA (http://www.cgga.org.cn/) and TCGA (http://cancergenome. nih.gov/). In CGGA dataset, 325 samples of RNA-seq data were collected into this study. To consolidate the findings that we have revealed in the CGGA dataset, RNA-seq data of 699 glioma samples of all grades from TCGA dataset were used as a validation cohort. Thus, in total, CD68 transcriptional expression data of 1,024 samples were evaluated.

\section{Statistical analyses}

The prognostic value of CD68 was estimated by KaplanMeier analysis. Other statistical computations and figure drawing were performed using several packages (ggplot2, pheatmap, pROC, and corrgram) in the statistical software environment R, version 3.4.1 (http://www.r-project. org). The main of $\mathrm{R}$ script is listed in the "Supplementary materials" section. For all statistical methods, $P<0.05$ was considered as a significant difference.

\section{Results \\ CD68 expression was upregulated in GBM and IDH wide-type glioma}

To obtain an overview of CD68 status in glioma, we first examined CD68 expression of RNA-seq data from the CGGA database. It turned out that CD68 was significantly upregulated in GBM (WHO IV) than that in WHO grade II and grade III gliomas (Figure 1A). Additionally, TCGA RNA-seq data were well validated to this result (Figure 1A). These results suggested that a higher CD68 expression was associated with a higher malignancy in gliomas. Emerging studies have demonstrated remarkably different clinical outcomes and oncogenic progression in glioma patients depending on the IDH-mutation status, which was added as a sub-classifier. ${ }^{17}$ Therefore, we further analyzed the relationship between CD68 expression and IDH-mutation status. We found that IDH wild-type (WT) glioma exhibited a higher level of CD68 than IDH-mutation glioma in the CGGA and TCGA datasets (Figure $1 \mathrm{~B})$. These data support the observation that upregulated CD68 may be more related to the neoplastic cell expansion and invasion of IDH WT glioma. Moreover, the performance of the CD68 expression in IDH WT patients was also performed by receiver operating characteristic (ROC) analyses. The areas under the curve (AUCs) were $70.5 \%$ and $72.7 \%$ in CGGA and TCGA datasets, respectively (Figure S1). Herein, CD68 may serve as a biomarker for IDH-mutant type glioma.

\section{CD68 expression was highly enriched in mesenchymal molecular subtype glioma}

To investigate the molecular expression pattern of CD68, we analyzed the distribution of CD68 in different molecular subtypes of glioma. ${ }^{18}$ As shown in Figure 2A, CD68 was significantly upregulated in mesenchymal subtype than that 
A

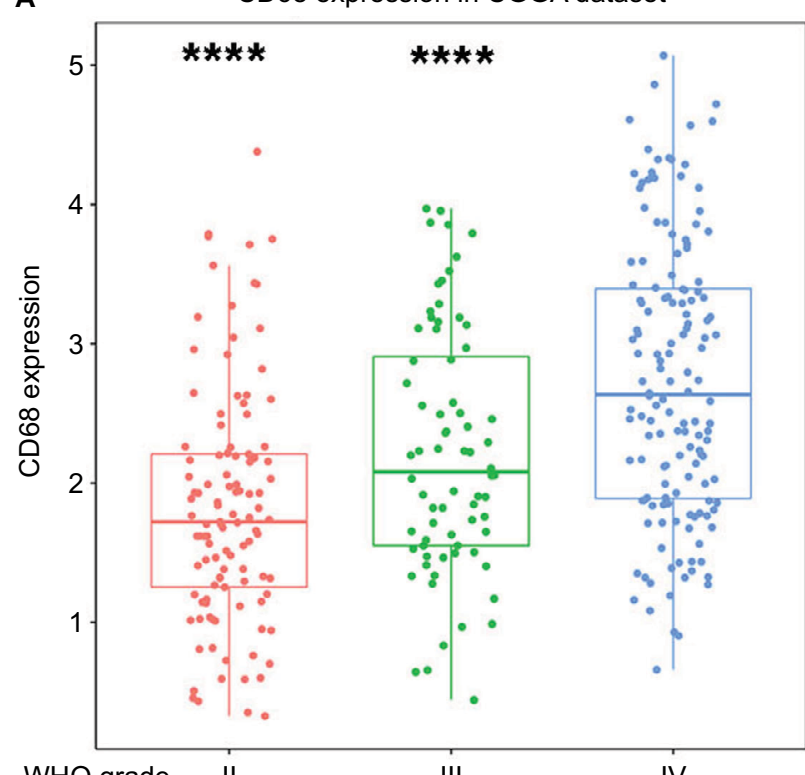

WHO grade II

B

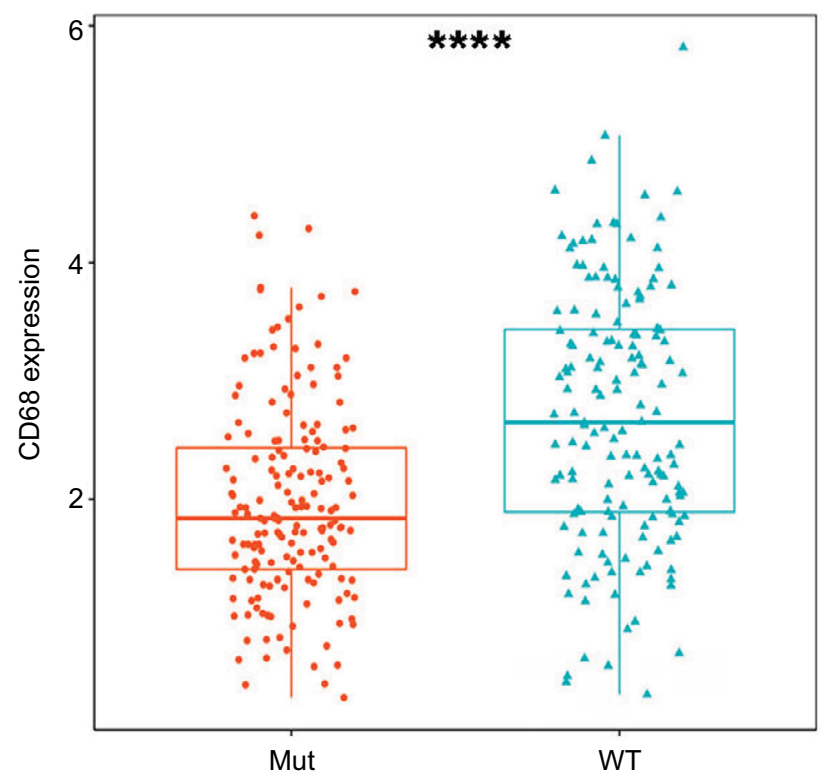

CD68 expression in TCGA dataset
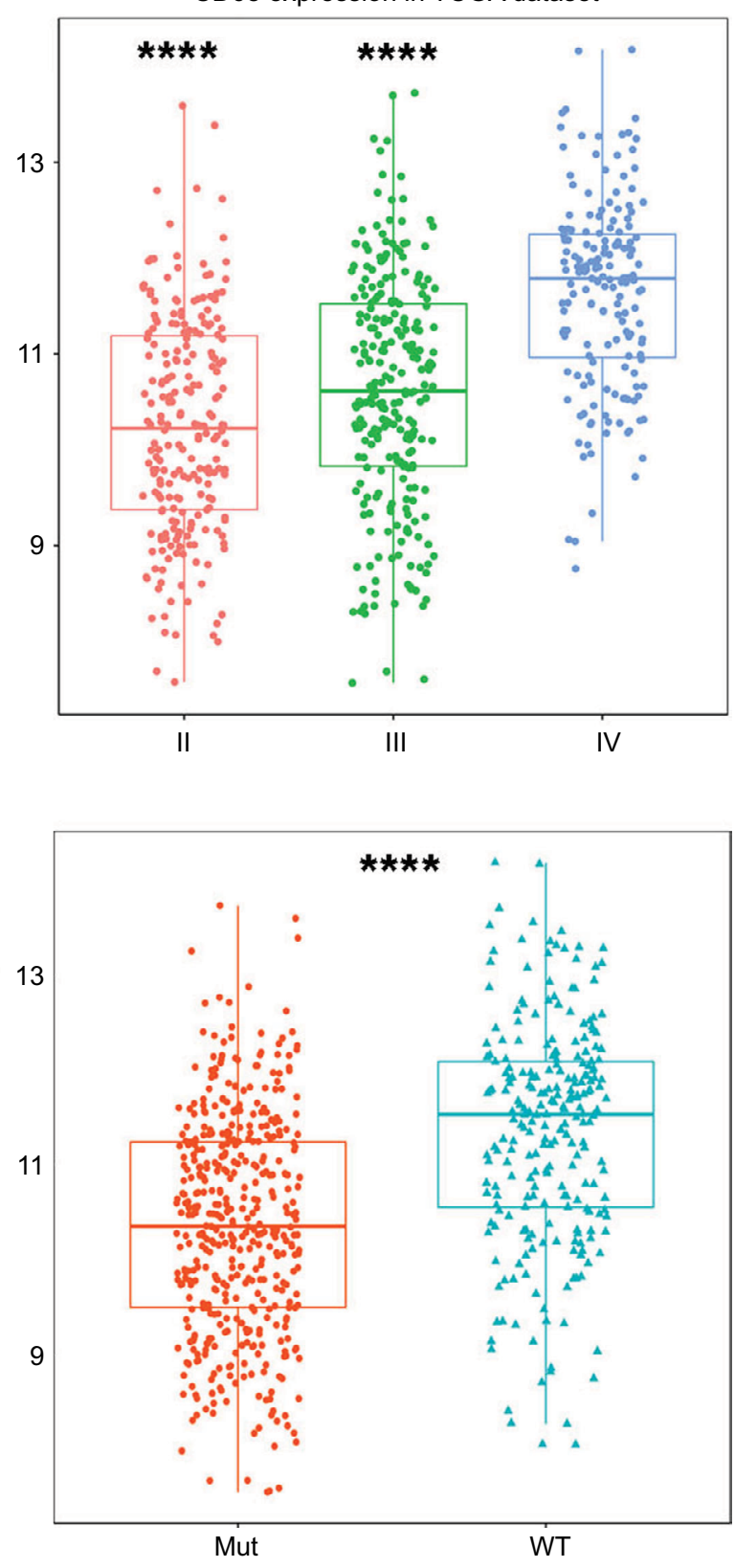

IDH mutation status

Figure I CD68 was highly expressed in GBM and IDH WT glioma.

Notes: (A) The mRNA level of CD68 was upregulated in GBM from CGGA and TCGA datasets. (B) CD68 was significantly increased in IDH WT glioma. **** $<0.000$ I.

Abbreviations: CGGA, Chinese Glioma Genome Atlas; GBM, glioblastoma; Mut, mutant; TCGA, The Cancer Genome Atlas; WT, wild type.

in other three subtypes in both CGGA and TCGA cohorts, indicating that $\mathrm{CD} 68$ might play an important role in different molecular subtypes. Furthermore, ROC curves for CD68 expression in mesenchymal subtype were performed, and the AUCs were $86.4 \%$ and $92.4 \%$ in CGGA and TCGA datasets, respectively (Figure 2B). These results showed that CD68 was highly expressed in mesenchymal-subtype glioma, which may play an important biologic function in glioma.

\section{CD68 expression was related to immune-related biological response}

The expression level of CD68 was heterogeneous in both different grades and molecular subtypes of glioma. Next, to gain insights into the contributions of CD68 expression to glioma biology, the gene ontology (GO) analysis in DAVID (https://david.ncifcrf.gov/) was used to screen relevant genes in CGGA and TCGA 
A

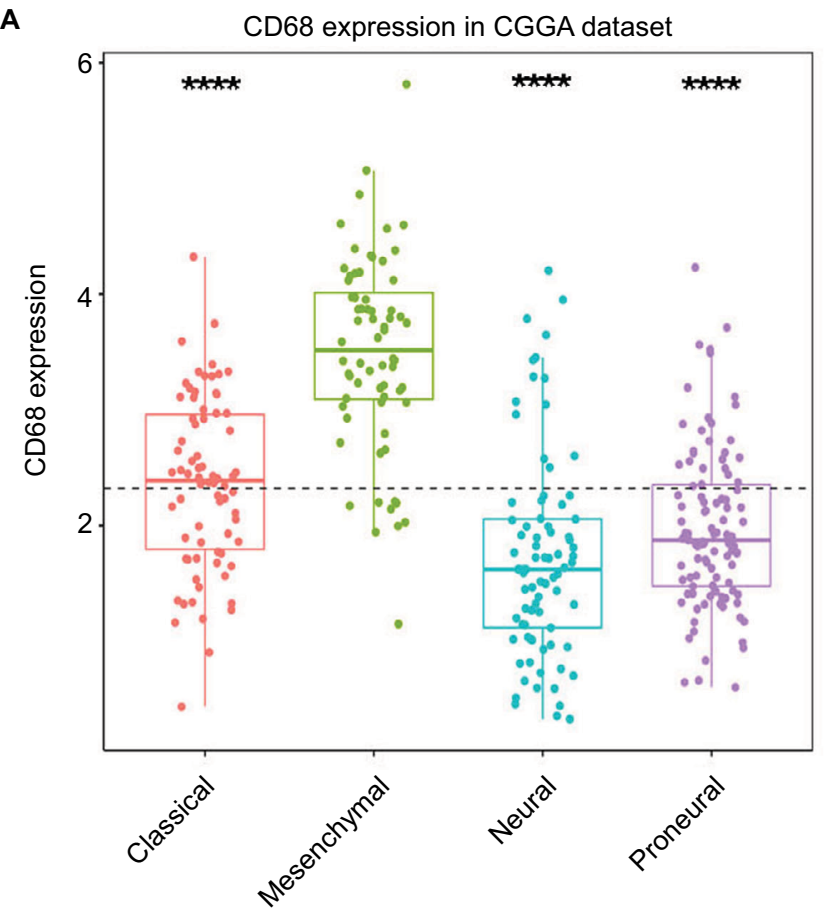

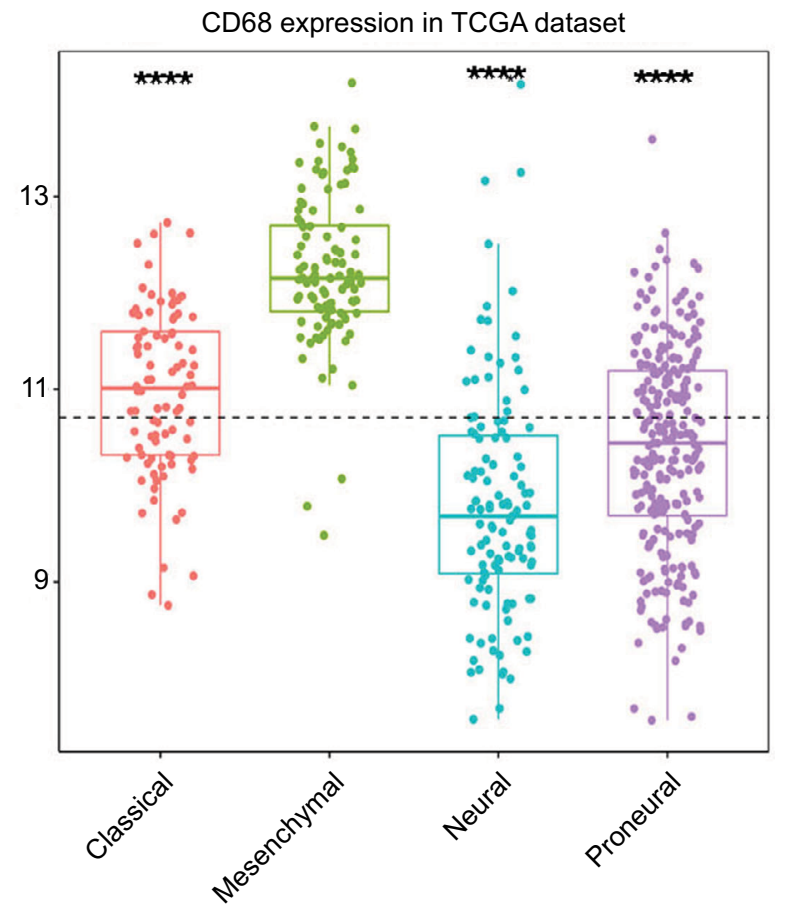

B

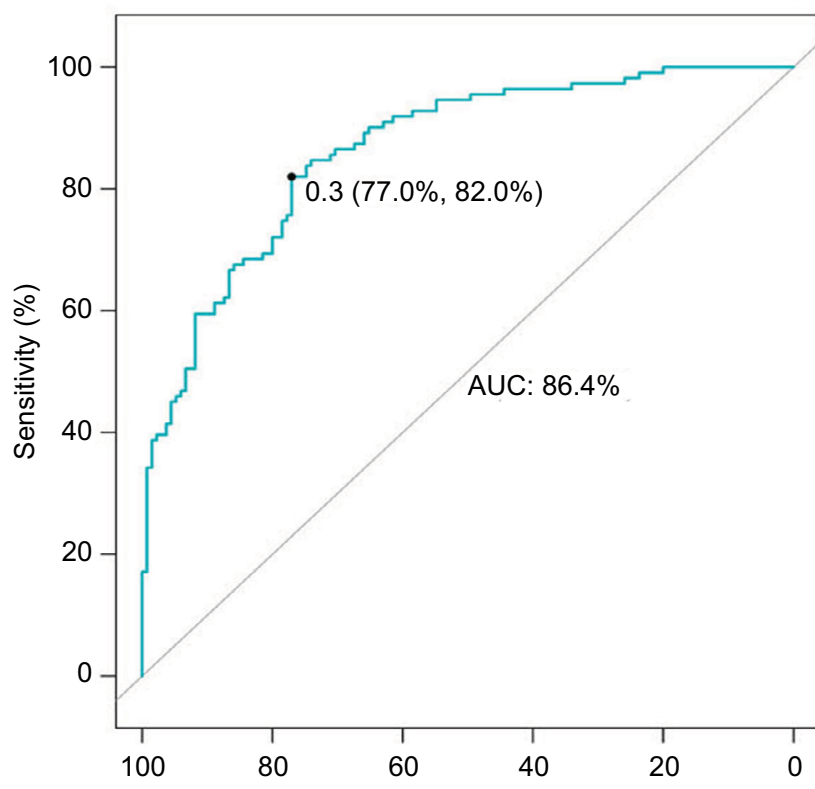

ROC curve

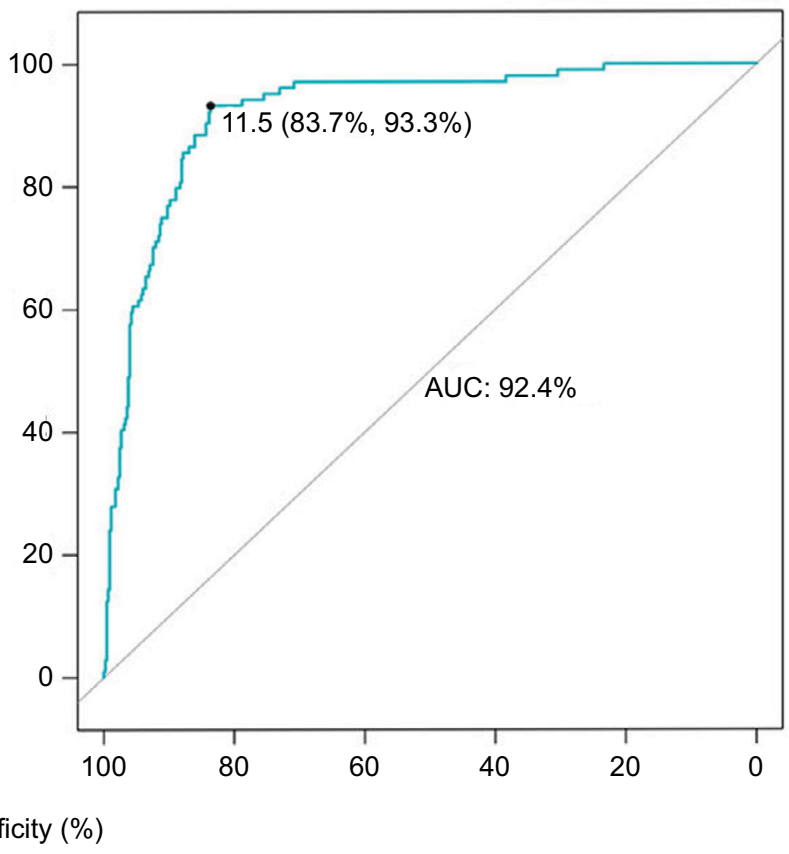

Figure 2 CD68 expression in four subtypes of glioma patients.

Notes: (A) CD68 was highly upregulated in mesenchymal molecular subtype. (B) CD68 could serve as a biomarker to predict mesenchymal subtype in CGGA and TCGA databases. $* * * * P<0.0001$.

Abbreviations: AUC, area under the curve; CGGA, Chinese Glioma Genome Atlas; ROC, receiver operating characteristic; TCGA, The Cancer Genome Atlas.

databases. The Pearson $|\mathrm{R}|$ value more than 0.4 was regarded as a significant correlation, and genes are shown in Figure $3 \mathrm{~A}$ and $\mathrm{B}$. We found that CD68 positively correlated genes were most involved in immune response, antigen processing and presentation, and T-cell costimulation in both CGGA and TCGA RNA-seq datasets (Figure 3A and B). The details of CD68 positively related immune process are shown in Figure $3 \mathrm{C}$ and $\mathrm{D}$, which were ranked by $P$-value. The genes most relevant to $\mathrm{CD} 68$ were mostly involved in inflammatory response, immune response, 
A

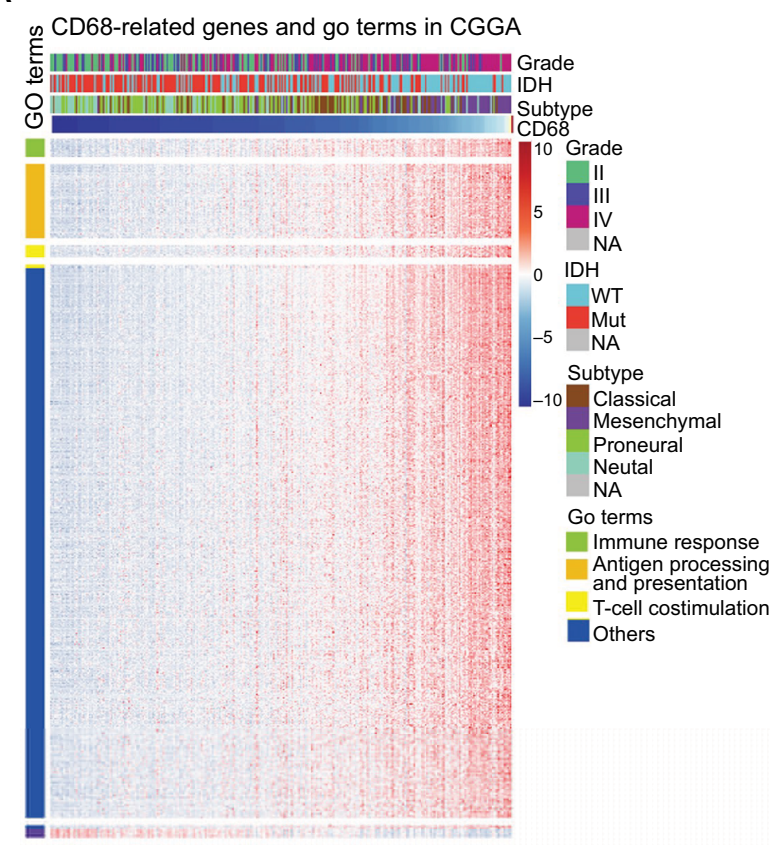

C

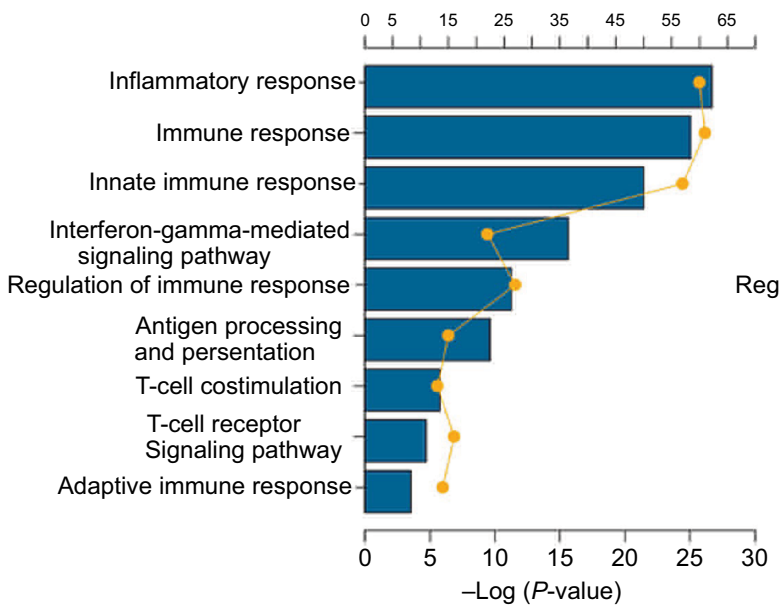

B

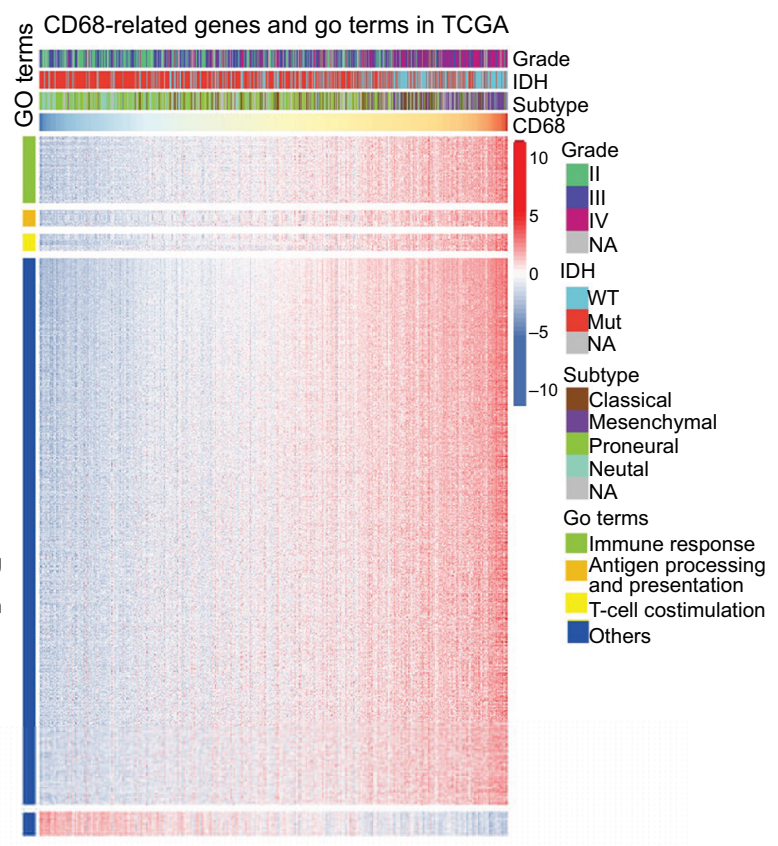

D

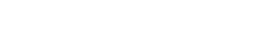

$\begin{array}{lllllllll}10 & 20 & 30 & 40 & 50 & 60 & 70 & 80 & 90\end{array}$

Inflammatory response

Innate immune response

Immune response

Interferon-gamma-mediated signaling pathway

Regulation of immune response

Antigen processing and persentation

Adaptive immune response

T-cell costimulation

T-cell receptor

Signaling pathway

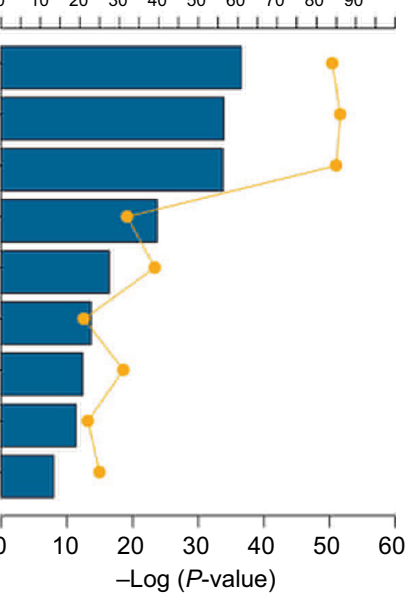

Figure 3 CD68 was closely related to immune response and inflammatory response in glioma.

Notes: (A and B) GO analysis exhibited that genes associated with CD68 expression were enriched in immune-related biological process. (C and D) The detail of CD68related immune process in CGGA and TCGA datasets.

Abbreviations: CGGA, Chinese Glioma Genome Atlas; GO, gene ontology; Mut, mutant; NA, not available; TCGA, The Cancer Genome Atlas; WT, wild type.

innate immune response, and interferon-gamma-mediated signaling pathway (Figure 3C and D). Taken together, these results suggested that $\mathrm{CD} 68$ played a key role in immune response and inflammatory response of glioma.

\section{Relationship between CD68 and inflammatory response}

Given the important role of CD68 in mediating inflammatory response, we further identified seven clusters of 104 inflammatory response-related genes, which were generated with results of Gene Set Variation Analysis (GSVA) and defined as metagenes. ${ }^{19}$ As shown in Figure $4 \mathrm{~A}$ and $\mathrm{B}$, in the CGGA dataset, CD68 expression was most correlated with the metagene of $\mathrm{HCK}$, indicating that CD68 was a specific marker for macrophages in glioma and displayed an immunosuppressive function. Meanwhile, CD68 also had a strong correlation with LCK, which was associated with T-cell activity. We observed a similar pattern in glioma of TCGA dataset (Figure S2). These results demonstrated that $\mathrm{CD} 68^{+}$ macrophages were upregulated as an immune suppressor when $T$ cells signaling transduction were activated in glioma.

\section{CD68 was synergistic with other markers of macrophages}

There is strong evidence of tumor progression by TAMs in glioma, and an increased prevalence of TAMs correlates with 


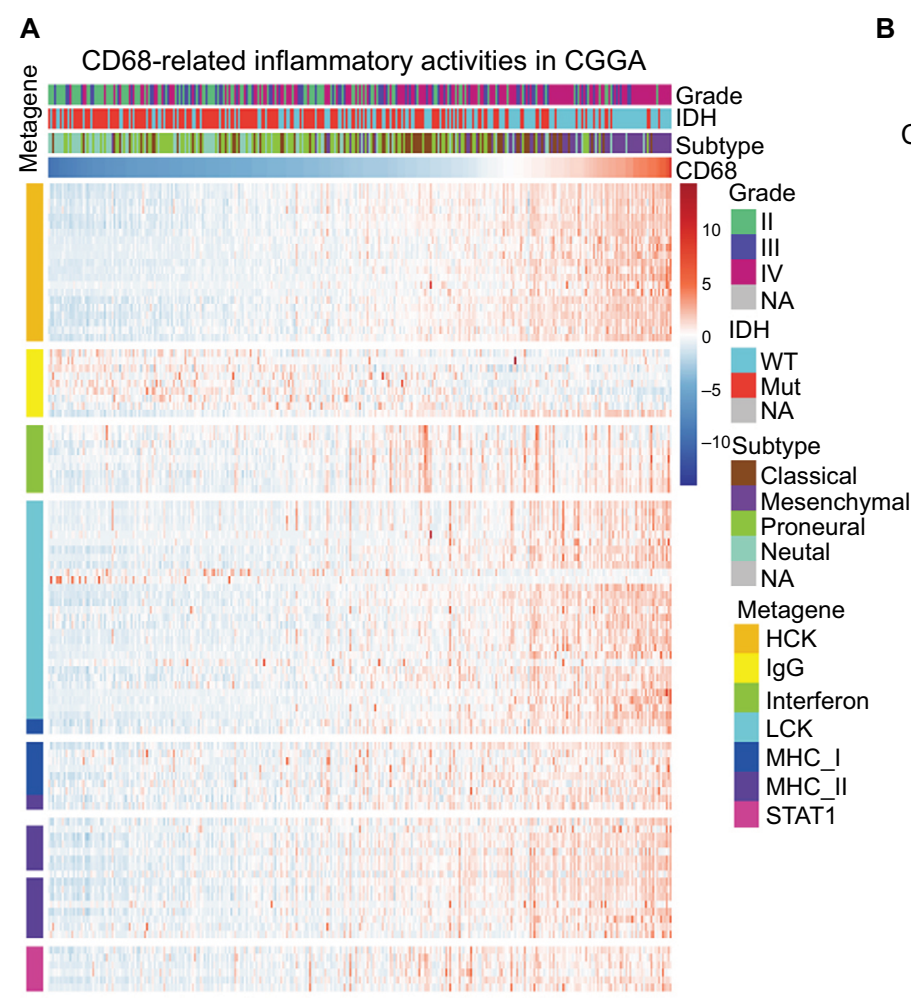

Figure 4 The relationship between CD68 and inflammatory metagenes.

Notes: (A) The heatmap of CD68-related inflammatory metagenes in the CGGA cohort. (B) Correlogram of CD68 and inflammatory metagenes in the CGGA cohort. Abbreviations: CGGA, Chinese Glioma Genome Atlas; Mut, mutant; NA, not available; WT, wild type.

short survival time.$^{20}$ Therefore, the surface markers of TAMs have been assessed as promise therapeutic targets. Moreover, TAMs are commonly identified by expression of CD68, CD11b, CD14, CD163, and CD204 in glioma. Herein, we put CD68 into analysis together with other markers. Pearson correlation was performed with these five factors in both CGGA and TCGA datasets. We observed that CD68 expression demonstrated high concordance with CD11b, CD14, CD163, and CD204, suggesting a synergistic relationship of these markers in glioma (Figure 5A and B). As we have seen, the tumor environment of GBM exhibited higher inflammation and immune response than that of low-grade glioma. To investigate the relationship among these markers of TAMs in GBM, Pearson correlation was also performed. As shown in Figure 5C and D, CD68 also had a robust correlation with other markers in CGGA and TCGA datasets. These results remind us that for patients who acquired resistance to immunotherapeutic markers based on the expression of CD68, we need to pay attention to the arising of other macrophage makers.

\section{Correlation between CD68 and immune checkpoints}

Agents targeting immune checkpoints are being extensively tested in preclinical or clinical trials for multiple solid tumors. ${ }^{21,22}$ Thus, checkpoint members were enrolled into the analysis, such as B7-H3, B7-H4, PD-1, LAG3, TIM3, and PD-L1. Surprisingly, CD68 showed strong positive correlations with PD-1, TIM-3, and B7-H3 in CGGA and TCGA datasets (Figure 6A and B). To our knowledge, PD-1 and TIM-3 seemed to express more in GBM and played almost exactly the same inflammatory activation functions in glioma. ${ }^{23,24}$ Further analysis revealed that CD68 showed a consistent correlation with PD-1 and TIM-3 in GBM (Figure 6C and D). It has been reported that PD-L1, B7-H3, and B7-H4 expression levels were associated with malignancy grade of glioma. ${ }^{25-27}$ Therefore, there was some relationship between CD68 and these immune checkpoints in whole-grade glioma. However, further analysis found that only PD-1 and TIM-3 showed a consistent relationship with CD68 in GBM. These results suggested that immunotherapeutic markers based on the expression of CD68 may be a great addition when patients acquire resistance to PD-1 or TIM- 3 inhibitors.

\section{CD68 expression was associated with poor survival}

As mentioned earlier, CD68 was an immunosuppressive maker and closely correlated with glioma progression. Thus, to validate the influence of CD68 on prognosis of 
A

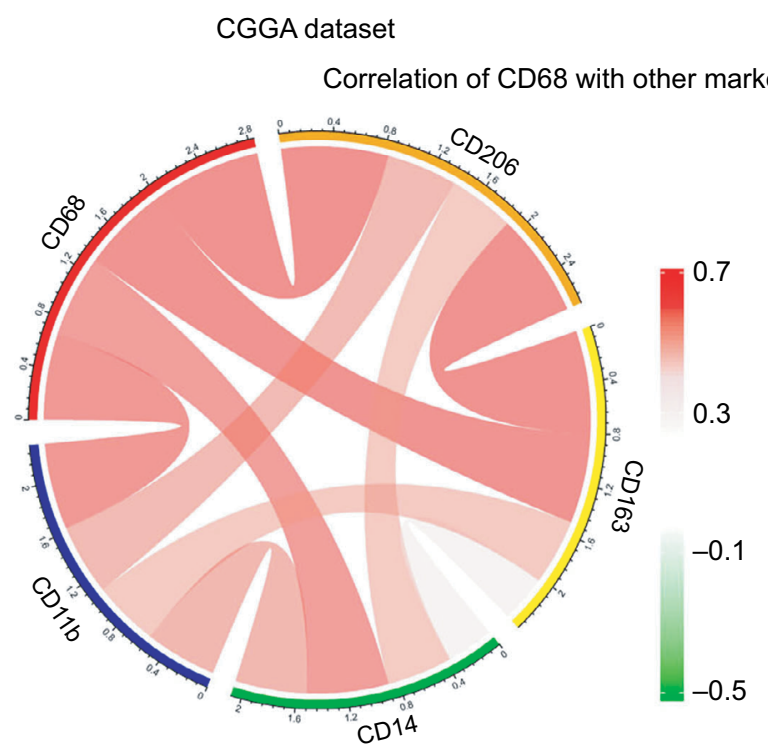

B

TCGA dataset

macrophages in glioma
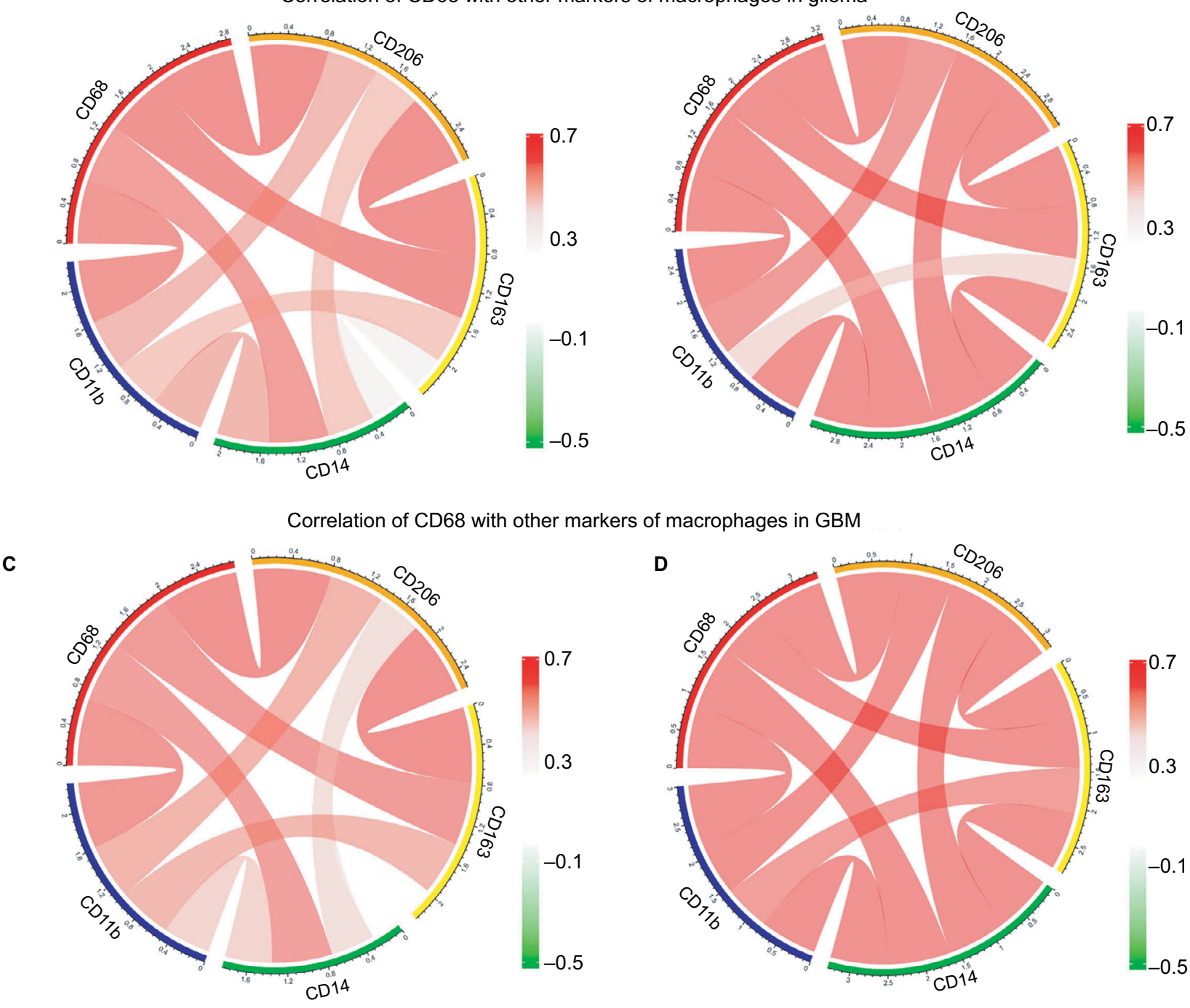

Figure 5 Relationship between CD68 and other markers of macrophages.

Notes: (A and B) Correlation of CD68 and other markers including CDI Ib, CDI4, CDI63, and CD206 in glioma from CGGA and TCGA datasets. (C and D) Correlation of CD68 and these makers in GBM.

Abbreviations: CGGA, Chinese Glioma Genome Atlas; GBM, glioblastoma; TCGA, The Cancer Genome Atlas.

glioma patients, we performed an overall survival (OS) analysis using the Kaplan-Meier method. Both in CGGA and TCGA datasets, compared with the low expression of CD68 group in whole-grade glioma, patients who had a higher CD68 expression had a significantly shorter survival (Figure 7A). Owing to heterogeneity across different grades of glioma, we further investigated the prognostic value of CD68 expression in GBM of two datasets. Interestingly, we observed the same prognostic tendency of CD68 in GBM patients (Figure 7B). Furthermore, the univariate Cox model of CD68 and other factors were analyzed, which also revealed that CD68 was tightly associated with OS $(P<0.001$; Table S1 $)$. However, the multivariate analysis showed that CD68 expression had no difference in survival (Table S1). These findings suggested that CD68 was a negative prognosticator in glioma due to the immunosuppressive effect of $\mathrm{CD} 68^{+}$macrophages.

\section{Discussion}

Glioma is the most common and lethal type of tumor in human brain. Tumor recurrence and treatment resistance are the results of both cancer cell growth and their interaction with tumor microenvironment. ${ }^{28}$ Recent studies showed that glioma tissues contain tumor cells and glioma-associated non-tumor cells, including stromal cells and immune cells. Notably, as an important type of 

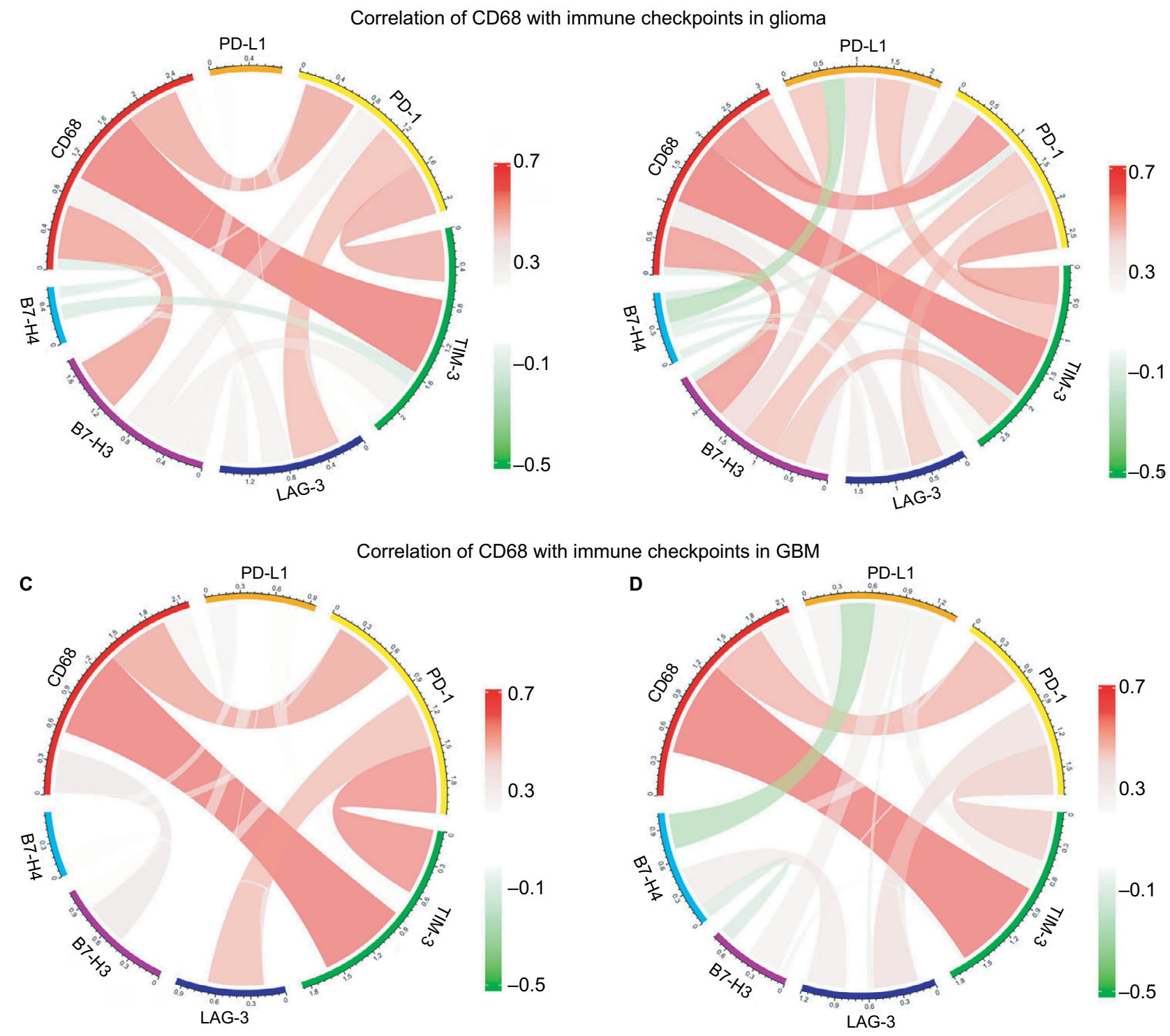

Figure 6 Correlation of CD68 and immune checkpoints.

Notes: (A and B) Relationship between CD68 and immune checkpoints in whole-grade glioma. (C and D) Correlation of CD68 and these immune checkpoints in GBM. Abbreviations: CGGA, Chinese Glioma Genome Atlas; GBM, glioblastoma; TCGA, The Cancer Genome Atlas.

immune cells, TAMs are abundant in glioma and act as a barrier to build an immunosuppressive microenvironment. ${ }^{29}$ Therefore, TAMs represent an important therapeutic target for cancer prevention and cure. The surface markers of TAMs such as CD68, CD163, CD11b, and CD206 can be directly targeted in the in vivo and in vitro experiments. ${ }^{10}$ In this study, we sought to carry out the largest and most comprehensive research of CD68 expression in glioma.

CD68 is a specific marker for M2-type macrophages and has an impact on the tumor progress. ${ }^{29}$ Previous studies examined the expression of CD68 in various types of cancer such as breast cancer, ovarian cancer, and astrocytomas. ${ }^{16,30,31}$ However, the landscape of CD68 expression in glioma remains unclear. Herein, the first and biggest integrative study, including 1,024 RNA-seq glioma patients from TCGA and CGGA datasets, characterizing CD68 expression in whole-grade glioma molecularly and clinically was carried out. As expected, CD68 was upregulated in higher malignant glioma, which was similar with the previous finding by Prosniak et al ${ }^{1}$ reported earlier. Furthermore, we also observed that CD68 was highly enriched in the phenotype of known malignant molecules such as IDH WT state, indicating that the IDH status may be involved 
A

CGGA dataset
TCGA dataset

Survival analysis of CD68 in glioma patients
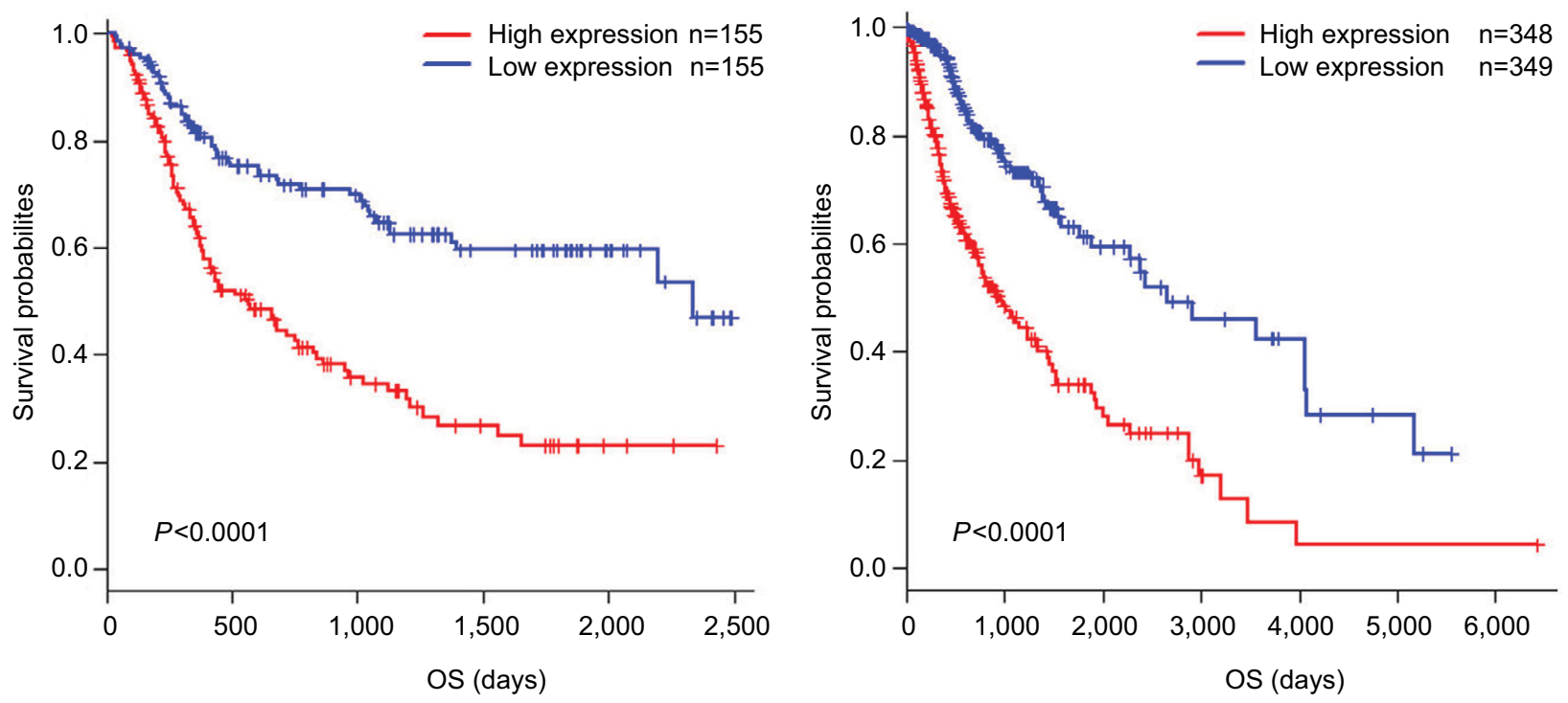

B Survival analysis of CD68 in GBM patients
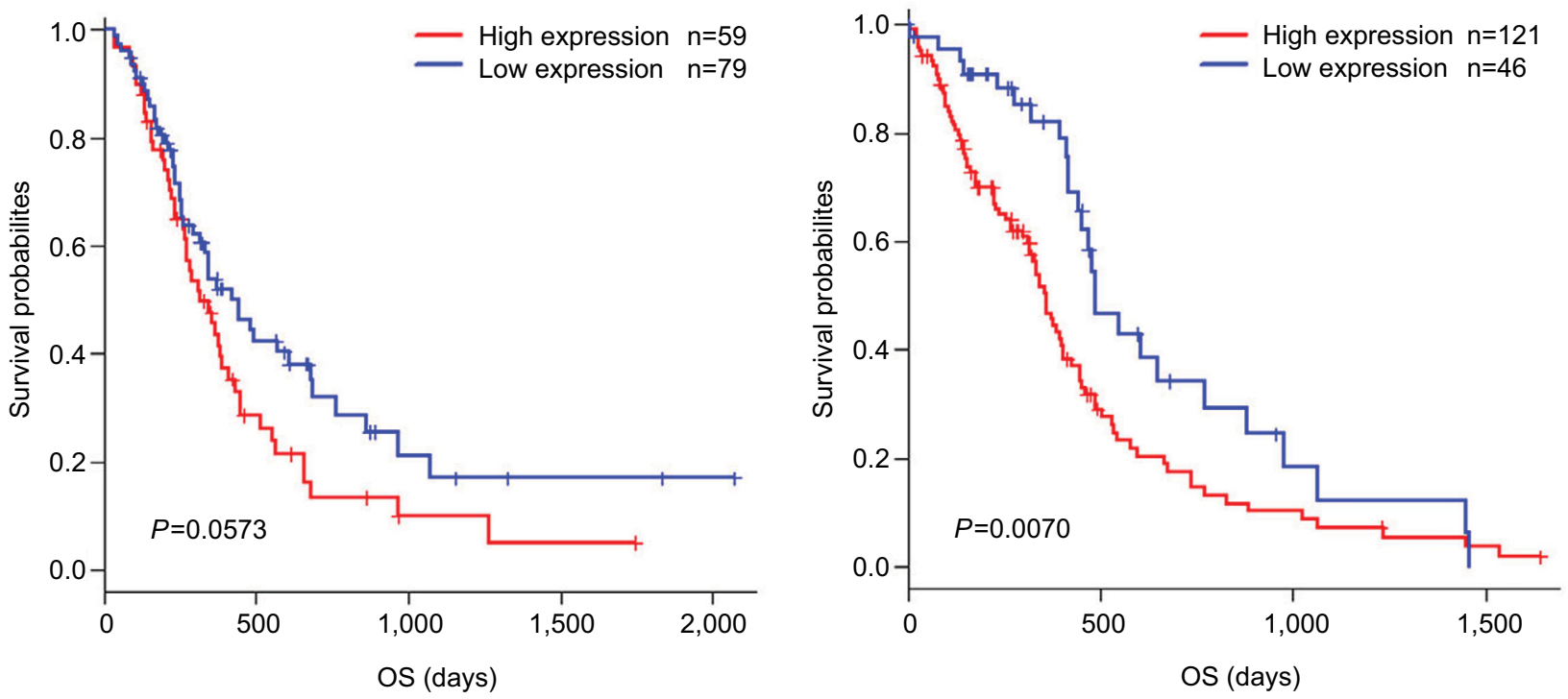

Figure 7 Survival analysis for CD68 expression in glioma patients.

Notes: (A and B) Survival analysis for CD68 expression in whole-grade glioma. (C and D) Survival analysis for CD68 in GBM.

Abbreviations: CGGA, Chinese Glioma Genome Atlas; GBM, glioblastoma; OS, overall survival; TCGA, The Cancer Genome Atlas.

in the immune processes of patients with glioma. The four molecular subtypes for glioma were proneural, neural, classical, and mesenchymal. The mesenchymal subtype is associated with poor survival and treatment resistance. ${ }^{32}$ Interestingly, we found that CD68 expressed significantly higher in mesenchymal subtype than that of other subtypes. Additionally, CD68 can be a biomarker for IDH wide type and mesenchymal phenotype. These results suggested that
CD68 was involved in the key step of immunosuppressive process for glioma.

We further investigated the CD68-related biology process in gliomas and found that genes closely related to CD68 were more involved in immune response and inflammatory response. In addition, seven clusters of metagenes were used to analyze the correlation between CD68 expression and inflammatory response. Results showed that CD68 had 
a strong correlation with $\mathrm{HCK}$ and LCK. In other words, CD68 was not only a specific marker for macrophages but also had a negative relationship with T-cell activation. It is well known that inflammation creates a microenvironment that causes neoplastic transformation and potentiates the progression of cancers. ${ }^{33}$ Thus, these results demonstrated that CD68 expression was tightly associated with tumorinduced immunosuppressive function.

Besides, the expression panel between CD68 and other markers of macrophages is still unknown. In glioma, the analysis of TAMs revealed a predominant population of $\mathrm{CD}_{163}{ }^{+}$and $\mathrm{CD}_{204^{+}}$anti-inflammatory M2 macrophages in association with higher tumor grade and a worse patient survival. ${ }^{11}$ The $\mathrm{CD} 11 \mathrm{~b}^{+}$and $\mathrm{CD} 14^{+}$cells were also accumulated in glioma with associated poor outcome. ${ }^{11}$ Based on the significance of CD163, CD204, CD11b, and CD14, we next analyzed the relationship between CD68 and these markers. The results showed that CD68 expression had a strong correlation with CD163, CD11b, CD14, and CD204, suggesting that CD68 and these markers played a synergistic role in tumor inflammatory response to promote tumor progression and metastasis. Emerging data showed that the development of monoclonal antibodies to block immune checkpoints is a promising treatment for glioma. ${ }^{34}$ Moreover, we analyzed the relationship between CD68 and immune checkpoints. Our results first revealed that CD68 showed a consistent synergy with PD-1 and TIM-3 in both TCGA and CGGA datasets. These results suggest that the combination of CD68 and immune checkpoint blockades may be feasible for glioma treatments. A previous study also showed that CD68 expression in glioma tissues may be used for prognosis of survival in these patients. ${ }^{35}$ Moreover, in both CGGA and TCGA datasets, it was verified that a higher CD68 expression level predicted significantly poor survival for glioma patients. This prognostic tendency was also observed in the patients with GBM. The significant prognostic significance of CD68 gives us more confidence that targeting CD68 significantly improves prognosis of glioma patients, especially GBM patients.

\section{Conclusion}

To our knowledge, this is the first study based on a large-scale glioma sample concerning CD68 analyses and its clinical significance. Although the exact mechanisms of CD68 in glioma still require further investigation, our study indicates that targeting CD68 has therapeutic promise for cancer immunotherapy based on its role as a macrophage-mediated immune suppression.

\section{Abbreviations}

TAMs, tumor-associated macrophages; CGGA, Chinese Glioma Genome Atlas; TCGA, The Cancer Genome Atlas; OS, overall survival; GO, gene ontology; GBM, glioblastoma; GSVA, Gene Set Variation Analysis

\section{Acknowledgment}

This study was supported by the National Key Research and Development Program of China (2016YFC1303501), Research Grant from the Ministry of Public Health (No. 201501004), and the Major Science and Technology Projects of Henan Province (No. 1611003101000).

\section{Author contributions}

All authors contributed to data analysis, drafting or revising the article, gave final approval of the version to be published, and agree to be accountable for all aspects of the work.

\section{Disclosure}

The authors report no conflicts of interest in this work.

\section{References}

1. Prosniak M, Harshyne LA, Andrews DW, et al. Glioma grade is associated with the accumulation and activity of cells bearing M2 monocyte markers. Clin Cancer Res. 2013;19(14):3776-3786.

2. Kanu OO, Mehta A, Di C, et al. Glioblastoma multiforme: a review of therapeutic targets. Expert Opin Ther Targets. 2009;13(6):701-718.

3. Grossman SA, Ye X, Piantadosi S, Desideri S, Nabors LB, Rosenfeld M, Fisher J; NABTT CNS Consortium. Survival of patients with newly diagnosed glioblastoma treated with radiation and temozolomide in research studies in the United States. Clin Cancer Res. 2010;16(8):2443-2449.

4. Quail DF, Bowman RL, Akkari L, et al. The tumor microenvironment underlies acquired resistance to CSF-1R inhibition in gliomas. Science. 2016;352(6288):aad3018.

5. Quail DF, Joyce JA. Microenvironmental regulation of tumor progression and metastasis. Nat Med. 2013;19(11):1423-1437.

6. Golebiewska A, Bougnaud S, Stieber D, et al. Side population in human glioblastoma is non-tumorigenic and characterizes brain endothelial cells. Brain. 2013;136(Pt 5):1462-1475.

7. Silver DJ, Sinyuk M, Vogelbaum MA, Ahluwalia MS, Lathia JD. The intersection of cancer, cancer stem cells, and the immune system: therapeutic opportunities. Neuro Oncol. 2016;18(2):153-159.

8. Poon CC, Sarkar S, Yong VW, Kelly JJP. Glioblastoma-associated microglia and macrophages: targets for therapies to improve prognosis. Brain. 2017;140(6):1548-1560.

9. Ruffell B, Coussens LM. Macrophages and therapeutic resistance in cancer. Cancer Cell. 2015;27(4):462-472.

10. Yang L, Zhang Y. Tumor-associated macrophages: from basic research to clinical application. J Hematol Oncol. 2017;10(1):58.

11. Lu-Emerson C, Snuderl M, Kirkpatrick ND, et al. Increase in tumorassociated macrophages after antiangiogenic therapy is associated with poor survival among patients with recurrent glioblastoma. Neuro Oncol. 2013;15(8):1079-1087.

12. Heusinkveld M, van der Burg SH. Identification and manipulation of tumor associated macrophages in human cancers. J Transl Med. 2011;9:216. 
13. Nywening TM, Wang-Gillam A, Sanford DE, et al. Targeting tumourassociated macrophages with CCR2 inhibition in combination with FOLFIRINOX in patients with borderline resectable and locally advanced pancreatic cancer: a single-centre, open-label, dose-finding, non-randomised, phase 1b trial. Lancet Oncol. 2016;17(5):651-662.

14. Yang L, Wang F, Wang L, et al. CD163+ tumor-associated macrophage is a prognostic biomarker and is associated with therapeutic effect on malignant pleural effusion of lung cancer patients. Oncotarget. 2015;6(12):10592-10603.

15. Rumney RMH, Coffelt SB, Neale TA, Dhayade S, Tozer GM, Miller G. PyMT-Maclow: a novel, inducible, murine model for determining the role of CD68 positive cells in breast tumor development. PLoS One. 2017;12(12):e0188591.

16. Ojalvo LS, Thompson ED, Wang TL, et al. Tumor-associated macrophages and the tumor immune microenvironment of primary and recurrent epithelial ovarian cancer. Hum Pathol. 2018;74:135-147.

17. Huse JT, Aldape KD. The evolving role of molecular markers in the diagnosis and management of diffuse glioma. Clin Cancer Res. 2014;20(22):5601-5611.

18. Verhaak RG, Hoadley KA, Purdom E, et al; Cancer Genome Atlas Research Network. Integrated genomic analysis identifies clinically relevant subtypes of glioblastoma characterized by abnormalities in PDGFRA, IDH1, EGFR, and NF1. Cancer Cell. 2010;17(1):98-110.

19. Rody A, Holtrich U, Pusztai L, et al. T-cell metagene predicts a favorable prognosis in estrogen receptor-negative and HER2-positive breast cancers. Breast Cancer Res. 2009;11(2):R15.

20. Roesch S, Rapp C, Dettling S, Herold-Mende C. When immune cells turn bad-tumor-associated microglia/macrophages in glioma. Int J Mol Sci. 2018;19(2):436.

21. Kim JE, Patel MA, Mangraviti A, et al. Combination therapy with antiPD-1, Anti-TIM-3, and focal radiation results in regression of murine gliomas. Clin Cancer Res. 2017;23(1):124-136.

22. Hugo W, Zaretsky JM, Sun L, et al. Genomic and transcriptomic features of response to anti-PD-1 therapy in metastatic melanoma. Cell. 2016;165(1):35-44.

23. Liu S, Wang Z, Wang Y, et al. PD-1 related transcriptome profile and clinical outcome in diffuse gliomas. Oncoimmunology. 2017;7(2):e1382792.
24. Li G, Wang Z, Zhang C, et al. Molecular and clinical characterization of TIM-3 in glioma through 1,024 samples. Oncoimmunology. 2017;6(8):e1328339.

25. Wang Z, Zhang C, Liu X, et al. Molecular and clinical characterization of PD-L1 expression at transcriptional level via 976 samples of brain glioma. Oncoimmunology. 2016;5(11):e1196310.

26. Baral A, Ye HX, Jiang PC, Yao Y, Mao Y. B7-H3 and B7-H1 expression in cerebral spinal fluid and tumor tissue correlates with the malignancy grade of glioma patients. Oncol Lett. 2014;8(3):1195-1201.

27. Yao Y, Ye H, Qi Z, et al. B7-H4(B7x)-mediated cross-talk between glioma-initiating cells and macrophages via the IL6/JAK/STAT3 pathway lead to poor prognosis in glioma patients. Clin Cancer Res. 2016;22(11):2778-2790.

28. Gieryng A, Pszczolkowska D, Walentynowicz KA, Rajan WD, Kaminska B. Immune microenvironment of gliomas. Lab Invest. 2017;97(5): 498-518.

29. Hambardzumyan D, Gutmann DH, Kettenmann H. The role of microglia and macrophages in glioma maintenance and progression. Nat Neurosci. 2016;19(1):20-27.

30. Morita Y, Zhang R, Leslie M, et al. Pathologic evaluation of tumorassociated macrophage density and vessel inflammation in invasive breast carcinomas. Oncol Lett. 2017;14(2):2111-2118.

31. Leenstra S, Das PK, Troost D, de Boer OJ, Bosch DA. Human malignant astrocytes express macrophage phenotype. J Neuroimmunol. 1995;56(1):17-25.

32. Sharma A, Bendre A, Mondal A, Muzumdar D, Goel N, Shiras A. Angiogenic gene signature derived from subtype specific cell models segregate proneural and mesenchymal glioblastoma. Front Oncol. 2017;7:146.

33. Pollard JW. Tumour-educated macrophages promote tumour progression and metastasis. Nat Rev Cancer. 2004;4(1):71-78.

34. Luksik AS, Maxwell R, Garzon-Muvdi T, Lim M. The role of immune checkpoint inhibition in the treatment of brain tumors. Neurotherapeutics. 2017;14(4):1049-1065

35. Strojnik T, Kavalar R, Zajc I, Diamandis EP, Oikonomopoulou K, Lah TT. Prognostic impact of CD68 and kallikrein 6 in human glioma. Anticancer Res. 2009;29(8):3269-3279.
Cancer Management and Research

\section{Publish your work in this journal}

Cancer Management and Research is an international, peer-reviewed open access journal focusing on cancer research and the optimal use of preventative and integrated treatment interventions to achieve improved outcomes, enhanced survival and quality of life for the cancer patient. The manuscript management system is completely online and includes

\section{Dovepress}

a very quick and fair peer-review system, which is all easy to use. Visit http://www.dovepress.com/testimonials.php to read real quotes from published authors. 